\title{
Article \\ Game-Theoretic Dynamic Procedure for a Power Index under Relative Symmetry
}

\author{
Jong-Chin Huang ${ }^{1}\left(\mathbb{D}\right.$, Kelvin H.-C. Chen ${ }^{1, *} \mathbb{C}$ and Yu-Hsien Liao ${ }^{2, *}$ \\ 1 Department of Applied Chemistry, National Pingtung University, Pingtung City 900391, Taiwan; \\ hj@@mail.nptu.edu.tw \\ 2 Department of Applied Mathematics, National Pingtung University, Pingtung City 900391, Taiwan \\ * Correspondence: kelvin@mail.nptu.edu.tw (K.H.-C.C.); twincos@ms25.hinet.net (Y.-H.L.)
}

Citation: Huang, J.-C.; Chen, K.H.-C.; Liao, Y.-H. Game-Theoretic Dynamic Procedure for a Power Index under Relative Symmetry. Symmetry 2021, 13, 1921. https:// doi.org/10.3390/sym13101921

Academic Editor: Dumitru Baleanu

Received: 8 September 2021

Accepted: 9 October 2021

Published: 13 October 2021

Publisher's Note: MDPI stays neutral with regard to jurisdictional claims in published maps and institutional affiliations.

Copyright: (c) 2021 by the authors. Licensee MDPI, Basel, Switzerland. This article is an open access article distributed under the terms and conditions of the Creative Commons Attribution (CC BY) license (https:// creativecommons.org/licenses/by/ $4.0 /)$.

\begin{abstract}
In many operational processes, a suitable combination of participating elements has a huge impact throughout the entire process. In the real environment, however, many combinations show less than expected results in the initial stage. In consideration of the many subjective and objective factors such as equipment, time, capital, materials, and so forth, it seems that the aforementioned combinations cannot be used to re-configure. It is important that these initial unsatisfactory combinations can gradually approach some equilibrium states or results through some rolling adjustment processes. In order to improve the above problem, this study attempts to use a game-theoretic dynamic procedure to establish a mechanism that can be dynamically modified under relative symmetry at any time during operational processes. Under such a dynamic procedure, an undesirable combination of participating elements can gradually approach a useful combination.
\end{abstract}

Keywords: operational processes; combination; relative symmetry; dynamic procedure

\section{Introduction}

In many modern academic studies, game theory is often applied to the equilibrium analysis of many operating systems. Through the participation degree or ratio between the participating factors, resource adjustment, behavior simulation, performance allocation, and process simulation are used to achieve the most balanced state. In brief, the axiomatic procedures in game theory often use various fields of mathematics to construct many concepts of fair and efficient distribution, and prove that these concepts of equilibrium also and only conform to some principles of fairness and justice, so as to analyze their mathematical correctness, application rationality, and actual acceptability. For instance, Shapley [1] introduced the Shapley value to analyze the utility-allocating situations by collecting the whole involvement expected value for each element. Hart and Mas-Colell [2] and Maschler and Owen [3] defined the self-reduction and the axioms of covariance, efficiency, standardness for games and symmetry to analyze the axiomatic results of the Shapley value, respectively. Ransmeier [4] defined the equal allocation non-separable costs (EANSC) as assigning the optimal output for dams managed by the Tennessee Valley Authority. By applying the disputing-conception of the EANSC, the elements first obtain their marginal contributions, and then allot the rest of profit equally. Moulin [5] defined the complement-reduction and the axioms of consistency, efficiency and equal treatment for equal and zero-independence to demonstrate that the EANSC is a steady allocation for sharing-profit.

The dynamic procedures in game theory are often used in various fields of mathematics to construct many rolling adjustment processes, so as to analyze some initial unsatisfactory states or results and to approach some equilibrium states or results dynamically through these processes. The foundation of a dynamic theory was laid by Stearns [6]. For example, by applying a specific reduction, Maschler and Owen [3] proposed a dynamic process to illustrate that the Shapley value can be reached by players who start from an arbitrary efficient payoff vector. Hwang [7] successfully adopted the excess function to 
provide a dynamic process leading to the EANSC. Related dynamic results could also be found in, for example, Billera [8], Hwang and Liao [9], Abuteen et al. [10], Al-Smadi [11], Freihet et al. [12] and so on.

Catalysts are always pondered to be essential elements under various chemical reaction processes. There are many distinct kinds of catalysts, such as chemical, enzymatic and photocatalytic catalysts. Although each participating catalyst could react under its characteristics, the efficiency of reacting interactions could be raised by a suitable combination of participating catalysts. By applying the game-theoretical outcomes to catalyticreacting environments, Chen et al. [13] introduced the multi-choice level-individual index (MLII) to analyze and derive the most efficient combinatorial mechanism for a collection of participating catalysts with different environments under multi-choice consideration. Further, Chen et al. [13] adopted the axiomatic procedures to show that the MLII is the only mechanism satisfying the properties of level completeness, the criterion for circumstances property, level equal effect property, level synchronization, and consonance simultaneously.

Motivated by the above results, we propose a dynamic procedure to reach the MLII for participating elements that start from a level complete combination and make consecutive amendments. Two main outcomes are as follows.

- In Section 3, we adopt the notion of surfeit to construct a modification mechanism. In brief, this mechanism collects the effect gap between a participating element and all other participating elements, and further adjusts the collected sum under relative symmetry, and finally uses it to correct the previous scale value. The notion of the relative symmetric modification mechanism is based on that of Maschler and Owen's [3] correction function and related dynamic outcome for the Shapley value. Some comparisons are also provided in Section 3.

- Furthermore, we show that any combination will be dynamically adjusted continuously through the above procedure, and it will gradually approach the MLII, reaching its limit.

\section{Preliminaries}

Let $U F$ be the universe of all potential elements. Any $p \in U F$ is said to be an element. For $p \in U F$ and $b_{p} \in \mathbb{N}, B_{p}=\left\{0,1, \cdots, b_{p}\right\}$ can be regarded as the active level space of element $p$ under an operational process, and $B_{p}^{+}=B_{p} \backslash\{0\}$, where 0 means no participation. Suppose that $F \subseteq U F$ is the grand collection of total participating elements of a specific operational process. Let $B^{F}=\prod_{p \in F} B_{p}$ be the product set of the active level spaces of all elements of $F$. Denote $0_{F}$ to be the zero vector under $\mathbb{R}^{F}$.

A multi-choice circumstance is denoted by $(F, b, C)$, where $F \neq \varnothing$ is a finite collection of elements, $b=\left(b_{p}\right)_{p \in F}$ is the vector that displays the maximal number of total acting levels for each element, and $C: B^{F} \rightarrow \mathbb{R}$ is an effect function with $C\left(0_{F}\right)=0$, which appoints to each $\lambda=\left(\lambda_{p}\right)_{p \in F} \in B^{F}$ the effect that the elements can produce when each element $p$ acts at active level $\lambda_{p}$. Denote the class of total multi-choice circumstances to be $\Psi$.

Let $(F, b, C) \in \Psi$ and $\lambda \in B^{F}$. Define $A(\lambda)=\left\{p \in F \mid \lambda_{p} \neq 0\right\}$ as the collection of the elements with acting non-zero levels, $\lambda_{K}$ to be the restriction of $\lambda$ at $K$ for every $K \subseteq F$, $\|\lambda\|=\sum_{p \in F} \lambda_{p}$ and $L^{F}=\left\{\left(p, k_{p}\right) \mid p \in F, k_{p} \in B_{p}^{+}\right\}$. A power index on $\Psi$ is a map $\tau$ assigning to each $(F, b, C) \in \Psi$ a vector

$$
\tau(F, b, C)=\left(\tau_{p, k_{p}}(F, b, C)\right)_{\left(p, k_{p}\right) \in L^{F}} \in \mathbb{R}^{L^{F}} .
$$

Here, $\tau_{p, k_{p}}(F, b, C)$ is the effect or the value of the element $p$ when it acts with level $k_{p}$ in $(F, b, C)$. For convenience, we define that $\tau_{p, 0}(F, b, C)=0$ for every $p \in F$.

In order to generate the most efficient combinations for a collection of catalysts, Chen et al. [13] introduced a power index under multi-choice circumstances as follows. 
Definition 1. The multi-choice level-individual index (MLII), $\bar{\gamma}$, is the map on $\Psi$ which associates to every $(F, b, C) \in \Psi$, every element $p \in F$ and every $k_{p} \in B_{p}^{+}$the effect:

$$
\bar{\gamma}_{p, k_{p}}(F, b, C)=\gamma_{p, k_{p}}(F, b, C)+\frac{1}{\|b\|} \cdot\left[C(b)-\sum_{t \in F} \sum_{q=1}^{b_{t}} \gamma_{t, q}(F, b, C)\right],
$$

where $\gamma_{p, k_{p}}(F, b, C)=C\left(k_{p}, 0_{F \backslash\{p\}}\right)-C\left(k_{p}-1,0_{F \backslash\{p\}}\right)$ is the individual-level distinction of $p \in F$ from level $k_{p}-1$ to $k_{p}$. Based on the power index $\bar{\gamma}$, elements first obtain their individuallevel distinctions under related levels, and further allot the rest of the effect among the whole active levels.

In order to present the dynamic procedure for the MLII, one should study some properties and related axiomatic outcomes. Let $\tau$ be a power index on $\Psi$.

- $\quad \tau$ satisfies level completeness (LCOM) if $\sum_{p \in F} \sum_{q=1}^{b_{p}} \tau_{p, q}(F, b, C)=C(b)$ for all $(F, b, C) \in \Psi$.

- $\tau$ satisfies the criterion for circumstances property (CFCP) if $\tau(F, b, C)=\bar{\gamma}(F, b, C)$ for all $(F, b, C) \in \Psi$ with $|F| \leq 2$.

- $\tau$ satisfies the level equal effect property (LEEP) if for all $(F, b, C) \in \Psi$ with $C\left(\lambda, k_{p}, 0\right)$ $C\left(\lambda, k_{p}-1,0\right)=C\left(\lambda, 0, k_{h}\right)-C\left(\lambda, 0, k_{h}-1\right)$ for some $\left(p, k_{p}\right),\left(h, k_{h}\right) \in L^{F}$ and for all $\lambda \in B^{F \backslash\{p, h\}}, \tau_{p, k_{p}}(F, b, C)=\tau_{h, k_{h}}(F, b, C)$.

- $\tau$ satisfies the level synchronization (LSYN) if for all $(F, b, C),(F, b, D) \in \Psi$ with $C(\lambda)=D(\lambda)+\sum_{p \in A(\lambda)} \sum_{q=1}^{\lambda_{p}} \mu_{p, q}$ for some $\mu \in \mathbb{R}^{L^{F}}$ and for all $\lambda \in B^{F}, \tau(F, b, C)=$ $\tau(F, b, D)+\mu$.

LCOM shows that total elements allot the whole utility completely when the total elements take the total levels in a circumstance. CFCP should be a self-sufficient situation if there exists only one element in the circumstance, but if there exist two elements in the circumstance, each of them first gains back what it could have yielded by itself, and they partake wholly in the rest of the profits and losses at the end of the circumstance. LEEP shows that the effects of two elements should be the same if the marginal distinctions of these two elements are coincidental. LSYN should be regarded to be an extreme weakness of the additivity. Given $(F, b, C) \in \Psi, H \subseteq F$ and a power index $\tau$, the reduced circumstance $\left(H, b_{H}, C_{H}^{\tau}\right)$ related to $H$ and $\tau$ is defined by, for all $\lambda \in B^{H}$,

$$
C_{H}^{\tau}(\lambda)= \begin{cases}0 & \lambda=0_{H}, \\ C\left(\lambda_{p}, 0_{F \backslash\{p\}}\right) & H \geq|2|, A(\lambda)=\{p\} \text { for some } p, \\ C\left(\lambda, b_{F \backslash H}\right)-\sum_{p \in F \backslash H} \sum_{q=1}^{b_{p}} \tau_{p, q}(F, b, C) & \text { otherwise. }\end{cases}
$$

For an arbitrary couple of elements under an environment, we consider a "reduced situation" among them by pondering the quantums remaining after the rest of the elements are given the effects stipulated by a power index $\tau$. Then, $\tau$ is known as the consonant if it continuously produces coincident effects as in the original environment, if it is employed in any reduced environment. A power index $\tau$ matches consonance (CSE) if for every $(F, b, C) \in \Psi,|F| \geq 3$, for every $H \subseteq F,|H|=2$ and for every $\left(p, k_{p}\right) \in L^{H}, \tau_{p, k_{p}}(F, b, C)=$ $\tau_{p, k_{p}}\left(H, b_{H}, C_{H}^{\tau}\right)$.

Remark 1. Two axiomatic outcomes are proposed by Chen et al. [13] as follows. Let $\tau$ be a power index.

- $\tau=\bar{\gamma}$ if and only if $\tau$ matches CSE and CFCP.

- $\tau=\bar{\gamma}$ if and only if $\tau$ matches CSE, LCOM, LEEP and LSYN. 


\section{Dynamic Procedure}

In this section, we introduce a dynamic procedure for the MLII. The basic concept of this revision adjustment is briefly described as follows: First, the participating element in a certain operational process seeks and collects the effect gap between a participating element and all other participating elements about whether each is used or not under relative symmetry, and then adjusts the collected sum by the a coefficient, and finally uses it to correct the previous scale value. In summary, the relationship between "the range of a coefficient" and "whether such a procedure can be close to MLII" is inseparable. That is, no matter what range the a coefficient is in, any level complement combination will be adjusted continuously through the above procedure, and finally it will be close to MLII. Therefore, the following results will state and examine this relationship.

Next, we adopt the concept of surfeit to provide a dynamic procedure that leads the elements to the MLII, beginning from a level complement combination. Some notations should be defined. Let $(F, b, C) \in \Psi$. The set of level complement outcomes on $(F, b, C)$ is defined as $L E(F, b, C)=\left\{x \in \mathbb{R}^{L^{F}} \mid \sum_{i \in F} \sum_{k_{i}=1}^{b_{i}} x_{i, k_{i}}=C(b)\right\}$ (If $b_{i}=1$ for all $i \in N$, then the level complement outcome coincides with the efficient outcome or the Pareto optimal outcome on traditional circumstances). Let $(F, b, C) \in \Psi$ and $x \in L E(F, b, C)$. The surfeit of a level vector $\lambda \in B^{F}$ at $x$ is:

$$
E C(\lambda, C, x)=\sum_{i \in F}\left[C(\lambda)-C\left(\lambda_{F \backslash\{i\}}, \lambda_{i}-1\right)-x_{i, \lambda_{i}}\right] .
$$

The value $E C(\lambda, C, x)$ can be regarded as the gap (or holler, variation) of $\lambda$ if the total elements obtain their effects from $x$ in $(F, b, C)$.

Based on Remark 1, the MLII is the only power index satisfying LCOM, LEEP, LSYN, and CSE. By considering the definitions of LEEP and surfeit simultaneously, an alternative viewpoint of the MLII could be generated as follows:

Lemma 1. Let $(F, b, C) \in \Psi$ and $x \in L E(F, b, C)$. Then,

$$
\begin{aligned}
& \left.E C\left(\left(k_{i}, 0_{F \backslash\{i\}}\right), C, x\right)=E C\left(\left(k_{j}, 0_{F \backslash\{j\}}\right), C, x\right)\right) \forall\left(i, k_{i}\right),\left(j, k_{j}\right) \in L^{F} \\
\Leftrightarrow \quad & x=\bar{\gamma}(N, v) .
\end{aligned}
$$

Proof. Let $(F, b, C) \in \Psi, x \in L E(F, b, C)$ and $\left(i, k_{i}\right) \in L^{F}$. Clearly,

$$
\begin{aligned}
& \sum_{j \in F \backslash\{i\}} \sum_{k_{j}=1}^{b_{j}}\left(E C\left(\left(k_{i}, 0_{F \backslash\{i\}}\right), C, x\right)-E C\left(\left(k_{j}, 0_{F \backslash\{j\}}\right), C, x\right)\right) \\
= & \sum_{j \in F \backslash\{i\}} \sum_{k_{j}=1}^{b_{j}}\left(C\left(k_{i}, 0_{F \backslash\{i\}}\right)-C\left(k_{i}-1,0_{F \backslash\{i\}}\right)-x_{i, k_{i}}-C\left(k_{j}, 0_{F \backslash\{j\}}\right)+C\left(k_{j}-1,0_{F \backslash\{j\}}\right)+x_{j, k_{j}}\right) \\
= & \sum_{j \in F \backslash\{i\}} \sum_{k_{j}=1}^{b_{j}}\left(\gamma_{i, k_{i}}(F, b, C)-x_{i, k_{i}}-\gamma_{j, k_{j}}(F, b, C)+x_{j, k_{j}}\right) \\
= & \sum_{j \in F \backslash\{i\}} \sum_{k_{j}=1}^{b_{j}}\left(\bar{\gamma}_{i, k_{i}}(F, b, C)-x_{i, k_{i}}-\bar{\gamma}_{j, k_{j}}(F, b, C)+x_{j, k_{j}}\right) \\
= & \|b\| \cdot\left(\bar{\gamma}_{i, k_{i}}(F, b, C)-x_{i, k_{i}}\right)+\sum_{j \in F} \sum_{k_{j}=1}^{b_{j}}\left(x_{j, k_{j}}-\bar{\gamma}_{j, k_{j}}(F, b, C)\right) \\
= & \|b\| \cdot\left(\bar{\gamma}_{i, k_{i}}(F, b, C)-x_{i, k_{i}}\right)+(C(b)-C(b)) \\
= & \|b\| \cdot\left(\bar{\gamma}_{i, k_{i}}(F, b, C)-x_{i, k_{i}}\right) .
\end{aligned}
$$


By Equation (4),

$$
\begin{aligned}
& \left.E C\left(\left(k_{i}, 0_{F \backslash\{i\}}\right), C, x\right)=E C\left(\left(k_{j}, 0_{F \backslash\{j\}}\right), C, x\right)\right) \forall\left(i, k_{i}\right),\left(j, k_{j}\right) \in L^{F} \\
\Leftrightarrow & 0=\sum_{j \in F \backslash\{i\}} \sum_{k_{j}=1}^{b_{j}}\left(E C\left(\left(k_{i}, 0_{F \backslash\{i\}}\right), C, x\right)-E C\left(\left(k_{j}, 0_{F \backslash\{j\}}\right), C, x\right)\right) \\
\Leftrightarrow & 0=\|b\| \cdot\left(\bar{\gamma}_{i, k_{i}}(F, b, C)-x_{i, k_{i}}\right) \\
\Leftrightarrow & \bar{\gamma}_{i, k_{i}}(F, b, C)=x_{i, k_{i}} \forall\left(i, k_{i}\right) \in L^{F} .
\end{aligned}
$$

Thus, $\bar{\gamma}(F, b, C)=x$.

Lemma 1 states that the level complement outcomes that achieve relative symmetry related to surfeit are coincident with the outcomes derived from the MLII. Based on Lemma 1, it is reasonable that a modification mechanism for arbitrary level complement outcomes could be considered by applying relative symmetry related to surfeit. Let $(F, b, C) \in \Psi, x \in L E(F, b, C)$ and $t>0$; we define the relative symmetric modification function $h: L E(F, b, C) \rightarrow \mathbb{R}^{L^{F}}$ as follows. $h=\left(h_{i, k_{i}}\right)_{\left(i, k_{i}\right) \in L^{F}}$ and for all $\left(i, k_{i}\right) \in L^{F}$,

$$
h_{i, k_{i}}(x)=x_{i, k_{i}}+t \cdot \sum_{j \in F \backslash\{i\}} \sum_{k_{j}=1}^{b_{j}}\left(E C\left(\left(k_{i}, 0_{F \backslash\{j\}}\right), C, x\right)-E C\left(\left(k_{j}, 0_{F \backslash\{i\}}\right), C, x\right)\right),
$$

where $t$ is a fixed positive scale, which makes known the assumption that element $i$ does not claim for full modification (if $t=1$ ) but only (often) for a fraction of it. The scale $t$ denotes how much the surfeit is altered. When elements participate in a circumstance, some surfeits from expectation may arise under different situations. The relative symmetric modification function is based on the idea that each element shortens the surfeit relating to its own and others' individual participation, and applies these regulations to amend the initial value.

A dynamic procedure mostly refers to a modification procedure in which unsatisfactory outcomes gradually trend towards satisfactory outcomes generated by a specific power index. The power indexes that produce satisfactory outcomes are generally examined by applying axiomatic characterization to analyze their uniqueness and verify that they satisfy fair, just, and widely accepted properties. Based on the aforementioned notion of dynamic procedures, we first evaluate the gaps between process involvement and outcomes, and adopted individual participatory behaviors, to define a set of surfeit concepts related to outcomes. Further, we apply relative symmetry related to surfeit and introduce a corresponding modification function to gradually adjust level complement outcomes to achieve relative symmetry related to surfeit. Lemma 1 indicated that the level complement outcomes that achieve relative symmetry related to surfeit are coincident with the outcomes derived from the MLII. By applying Remark 1, the level complement outcomes that achieve relative symmetry related to surfeit also satisfy LCOM, LEEP, LSYN, and CSE.

The following result shows that the relative symmetric modification function is welldefined. This result also plays an essential role in proving our convergence result.

Lemma 2. $h(x) \in L E(F, b, C)$ for all $(F, b, C) \in \Psi$ and $x \in L E(F, b, C)$.

Proof. Let $(F, b, C) \in \Psi, x \in L E(F, b, C)$ and $\left(i, k_{i}\right) \in L^{F}$. By Equation (4),

$$
\sum_{j \in F \backslash\{i\}} \sum_{k_{j}=1}^{b_{j}}\left(E C\left(\left(k_{i}, 0_{F \backslash\{i\}}\right), C, x\right)-E C\left(\left(k_{j}, 0_{F \backslash\{j\}}\right), C, x\right)\right)=\|b\| \cdot\left(\bar{\gamma}_{i, k_{i}}(F, b, C)-x_{i, k_{i}}\right) .
$$

Hence, by Equations (6) and (7), 


$$
\begin{aligned}
& \sum_{i \in F} \sum_{k_{i}=1}^{b_{i}} h_{i, k_{i}}(x) \\
= & \sum_{i \in F} \sum_{k_{i}=1}^{b_{i}}\left[x_{i, k_{i}}+t \cdot \sum_{j \in F \backslash\{i\}} \sum_{k_{j}=1}^{b_{j}}\left(E C\left(\left(k_{i}, 0_{F \backslash\{j\}}\right), C, x\right)-E C\left(\left(k_{j}, 0_{F \backslash\{i\}}\right), C, x\right)\right)\right] \\
= & \sum_{i \in F} \sum_{k_{i}=1}^{b_{i}} x_{i, k_{i}}+t \cdot \sum_{i \in F} \sum_{k_{i}=1}^{b_{i}}\left[\sum_{j \in F \backslash\{i\}} \sum_{k_{j}=1}^{b_{j}}\left(E C\left(\left(k_{i}, 0_{F \backslash\{j\}}\right), C, x\right)-E C\left(\left(k_{j}, 0_{F \backslash\{i\}}\right), C, x\right)\right)\right] \\
= & C(b)+t \cdot\|b\| \sum_{i \in F} \sum_{k_{i}=1}^{b_{i}}\left(\bar{\gamma}_{i, k_{i}}(F, b, C)-x_{i, k_{i}}\right) \\
= & C(b)+t \cdot\|b\|(C(b)-C(b)) \\
= & C(b) .
\end{aligned}
$$

Thus, $h(x) \in \operatorname{LE}(F, b, C)$.

Remark 2. By Equations (4) and (6), it is easy to have that $h(\bar{\gamma}(F, b, C))=\bar{\gamma}(F, b, C)$ for all $(F, b, C) \in \Psi$.

Let $(F, b, C) \in \Psi$ and $x \in \operatorname{LE}(F, b, C)$. We define the dynamic sequences $\left\{x^{q}\right\}_{q=1}^{\infty}$ to be that $x^{0}(F, b, C)=x, \cdots, x^{q}=h\left(x^{q-1}\right)$ for all $q \in \mathbb{N}$.

Theorem 1. If $0<t<\frac{2}{\|b\|}$, then $\left\{x_{i, k_{i}}^{q}\right\}_{q=1}^{\infty}$ converges to $\bar{\gamma}_{i, k_{i}}(F, b, C)$ for all $(F, b, C) \in \Psi$, for all $x \in L E(F, b, C)$ and for all $\left(i, k_{i}\right) \in L^{F}$.

Proof. Let $(F, b, C) \in \Psi$ and $x \in L E(F, b, C)$. By the definition of $h$ and Lemma 2, for every $\left(i, k_{i}\right) \in L^{F}$,

$$
\begin{aligned}
h_{i, k_{i}}(\tau(F, b, C))-x_{i, k_{i}} & =t \cdot \sum_{j \in F \backslash\{i\}} \sum_{k_{j}=1}^{b_{j}}\left(E C\left(\left(k_{i}, 0_{F \backslash\{j\}}\right), C, x\right)-E C\left(\left(k_{j}, 0_{F \backslash\{i\}}\right), C, x\right)\right) \\
& =t \cdot\|b\| \cdot\left(\bar{\gamma}_{i, k_{i}}(F, b, C)-x_{i, k_{i}}\right) .
\end{aligned}
$$

Hence,

$$
\begin{aligned}
\bar{\gamma}_{i, k_{i}}(F, b, C)-h_{i, k_{i}}(x) & =\bar{\gamma}_{i, k_{i}}(F, b, C)-x_{i, k_{i}}+x_{i, k_{i}}-h_{i, k_{i}}(\tau(F, b, C)) \\
& =\bar{\gamma}_{i, k_{i}}(F, b, C)-x_{i, k_{i}}-t \cdot\|b\| \cdot\left(\bar{\gamma}_{i, k_{i}}(F, b, C)-x_{i, k_{i}}\right) \\
& =(1-t \cdot\|b\|) \cdot\left[\bar{\gamma}_{i, k_{i}}(F, b, C)-x_{i, k_{i}}\right] .
\end{aligned}
$$

So, for all $q \in \mathbb{N}, \bar{\gamma}_{i, k_{i}}(F, b, C)-x_{i, k_{i}}^{q}=(1-t \cdot\|b\|)^{q} \cdot\left[\bar{\gamma}_{i, k_{i}}(F, b, C)-x_{i, k_{i}}\right]$. If $0<t<$ $\frac{2}{\|b\|}$, then $-1<(1-t \cdot\|b\|)<1$ and $\left\{x_{i, k_{i}}^{q}\right\}_{q=1}^{\infty}$ converges geometrically to $\bar{\gamma}_{i, k_{i}}(F, b, C)$.

Inspired by Maschler and Owen [3], we would like to define a specific reduction to exhibit a different dynamic procedure. An extension of the specific reduction and related correction function due to Maschler and Owen [3] could be defined as follows:

- $\quad$ Let $(F, b, C) \in \Psi, H \subseteq F$ and $x \in L E(F, b, C)$. The specific reduced circumstance $\left(H, b_{H}, C_{H}^{\bar{\gamma}, x}\right)$ is defined by:

$$
C_{H}^{\bar{\gamma}, x}(\lambda)= \begin{cases}C\left(\lambda, b_{F \backslash H}\right)-\sum_{p \in F \backslash H} \sum_{q=1}^{b_{p}} x_{p, q} & , \lambda=b_{H}, \\ C_{H}^{\bar{\gamma}}(\lambda) & , \text { otherwise. }\end{cases}
$$

For all $\lambda \in B^{H}$. If $x=\bar{\gamma}(F, b, C)$, then $C_{H}^{\bar{\gamma}, x}=C_{H}^{\bar{\gamma}}$ for all $H \subseteq F$. 
- $\quad$ Let $(F, b, C) \in \Psi, x \in L E(F, b, C)$ and $t>0$, we define the correction function $g$ : $L E(F, b, C) \rightarrow \mathbb{R}^{L^{F}}$ as follows. $g=\left(g_{i, k_{i}}\right)_{\left(i, k_{i}\right) \in L^{F}}$ and for all $\left(i, k_{i}\right) \in L^{F}$,

$$
g_{i, k_{i}}(x)=x_{i, k_{i}}+t \cdot \sum_{j \in F \backslash\{i\}} \sum_{q=1}^{b_{i}}\left(\bar{\gamma}_{i, q}\left(\{i, j\}, b_{\{i, j\}}, C_{\{i, j\}}^{\bar{\gamma}, x}\right)-x_{i, q}\right) \text {. }
$$

Let $(F, b, C) \in \Psi$ and $y \in L E(F, b, C)$. We define the dynamic sequences $\left\{y^{q}\right\}_{q=1}^{\infty}$ to be that $y^{0}(F, b, C)=y, \cdots, y^{q}=g\left(y^{q-1}\right)$ for all $q \in \mathbb{N}$. Similar to the proofs of Maschler and Owen [3] and Theorem 1, it is easy to have the following dynamic result.

Theorem 2. If $0<t<\frac{4}{\|b\|}$, then $\left\{y_{i, k_{i}}^{q}\right\}_{q=1}^{\infty}$ converges to $\bar{\gamma}_{i, k_{i}}(F, b, C)$ for all $(F, b, C) \in \Psi$, for all $y \in L E(F, b, C)$ and for all $\left(i, k_{i}\right) \in L^{F}$.

Remark 3. The correction function is based on the idea that each element finds all the others to participate again on the basis of the specific reduction if the initial outcome does not satisfy entire elements. Further, the MLII is applied to redistribution, to determine the difference from the initial outcome. Finally, these regulations are used to correct the initial value. The major differences are as follows:

- The relative symmetric modification mechanism is based on "surfeit", and the correction function is based on "reduction";

- The convergent intervals related to these two dynamic results are different.

\section{Discussion and Application}

The combinations of elements may be very important under whole operational processes. In the past, combinations were always estimated by empirical rule judgments, literature comparison simulations, circular errata corrections and so on. Although the MLII has been shown to be able to propose a relatively balanced combination ratio for all reacting processes (please see Chen et al. [13]), as stated in previous sections, not all experimental procedures will adopt the MLII to analyze the combinations of catalysts at the beginning. On the other hand, although all the participating elements have their own characteristics during the operational processes, they may produce mutual excitation, repulsion, or other interactive effects between each other, which will have a relative impact on the processes and results of entire operational processes. Therefore, with such inspiration, a dynamic procedure is proposed in this research.

In order to demonstrate how the notions of multi-choice circumstance, the MLII and related dynamic procedure could be exploited and to make the implications clearer, we offer an example of reacting processes.

Example 1. To evaluate the usefulness of the combination of antidotes and catalysts, empiric prognostication, inference of posterior outcomes, or simulation of similar programs, as well as the construction, simulation and derivation of theoretical results in distinct spheres, may be conducted to examine the exactness, suitability, validity and reasonability of such combinations. The scientific community is capitalizing on many innovations to transform traditional approaches used for numerous toxic mitigation measures under aquatic environments. Related investigations have been introduced, such as Habschied [14], Mouchbahani-Constance et al. [15], Peles et al. [16], Reichwaldt et al. [17], Sotnichenko et al [18], and so on.

In the following, an application for toxic mitigation measures under aquatic environments is provided. Suppose that $F$ is the collection of heavy metal pollutants and mitigating catalysts. In real-world situations, however, allocation, domination, regulation and imitation always vary comparatively to each other in response to the abruptly altering interplay among elements, flocks, and circumstances. Hence, let the capacity of the reacting levels of every $p \in F$ be $B_{p}$. A reacting level vector $\lambda \in B^{F}$ could be regarded as a combination of reacting levels for mitigating catalysts and heavy metal pollutants meant to determine some effects or impacts, which are coincident with 
its elements. The synergistic effects of a reacting level vector $\lambda$ of mitigating catalysts and pollutants after the application of antioxidants and metal chelators (i.e., $C(\lambda)$ ) are determined under the mode that mitigating catalysts and pollutants exert multiplex styles of reacting levels simultaneously. By applying the notion of multi-choice circumstance, a toxicity-reacting environment with lofty consistences of mitigating catalysts and heavy metal pollutants can be generalized as $(F, b, C)$. Based on the works of Chen et al. [13], it is shown that the MLII could be a stable and sustainable mechanism for generating the most efficient combinations for reacting levels among all elements.

To evaluate the effect of each element, we first assess the individual-level effect each operational element has accumulated over reacting processes based on various and distinct levels, which is the the individual-level distinction $\gamma$ mentioned in Definition 1. The remaining generated efficacy distribution should also be allocated, entirely derived for each element and its reactive levels, which is the MLII $\bar{\gamma}$ mentioned in Definition 1. In some real environments, however, many combinations derived from other methods show less than expected results in the initial stage under reacting processes. Let $x=\left(x_{p, k_{p}}\right) \in L E(F, b, C)$ be an arbitrary level complement combination for reacting levels among all elements. By applying the dynamic outcome of this article, $x$ can be continuously adjusted by means of relative symmetric modification function $h$ under the reacting processes, and finally it will gradually be close to the balanced combination $\bar{\gamma}(F, b, C)$ generated by the MLII.

Next, a numerical appliance is offered. Let $(F, b, C) \in \Psi$ be an aquatic environment with mitigating catalysts and heavy metal pollutants set $A=\{p, q, k\}$ and reacting level vector $b=(2,1,1)$. Let $C(1,0,0)=-1, C(2,0,0)=9, C(0,1,0)=2, C(1,1,0)=2$, $C(0,0,1)=-3, C(1,0,1)=-7, C(0,1,1)=3, C(1,1,1)=4, C(2,0,1)=5, C(2,1,0)=6$, $C(2,1,1)=5$ and $C(0,0,0)=0$ be the effect that the elements can elicit under whole reacting processes. Thus,

$$
\begin{aligned}
& \gamma_{p, 2}(F, b, C)=10, \quad \gamma_{p, 1}(F, b, C)=-1, \\
& \gamma_{q, 1}(F, b, C)=2, \quad \gamma_{k, 1}(F, b, C)=-3, \\
& \overline{\gamma_{p, 2}}(F, b, C)=9.25, \quad \overline{\gamma_{p, 1}}(F, b, C)=-1.75, \\
& \gamma_{q, 1}(F, b, C)=1.25, \quad \frac{\gamma_{k, 1}}{\gamma_{1}}(F, b, C)=-3.75 .
\end{aligned}
$$

Clearly, the effect of each element when it reacts with a reacting level is $(F, b, C)$. For instance, the effect of element $k$ is $\overline{\gamma_{k, 1}}(F, b, C)=-3.75$ if $k$ reacts with the level 1 in $(F, b, C)$.

Let $x=\left(x_{p, 2}, x_{p, 1}, x_{q, 1}, x_{k, 1}\right)=(7,-3,-1,2) \in L E(F, b, C)$ be an arbitrary level complement combination for reacting levels among all elements. Suppose that $x^{0}=x$. Clearly, $E C\left((2,0,0), C, x^{0}\right)=C(2,0,0)-C(1,0,0)-x_{p, 2}^{0}=3$. Similarly, $E C\left((1,0,0), C, x^{0}\right)=2$, $E C\left((0,1,0), C, x^{0}\right)=3, E C\left((0,0,1), C, x^{0}\right)=-5$. Let $t=\frac{1}{4}$. By Equation (1),

$$
\begin{aligned}
x_{p, 2}^{1} & =h_{p, 2}\left(x^{0}\right) \\
& =x_{p, 2}^{0}+t \cdot\left[\left(E C\left((2,0,0), C, x^{0}\right)-E C\left((0,1,0), C, x^{0}\right)\right)+\left(E C\left((2,0,0), C, x^{0}\right)-E C\left((0,0,1), C, x^{0}\right)\right)\right] \\
& =7+\frac{1}{4} \cdot[(3-3)+(3-(-5))] \\
& =9 .
\end{aligned}
$$

Similarly, $x_{p, 1}^{1}=-1.5, x_{q, 1}^{1}=1.25, x_{k, 1}^{1}=-3.75$, that is, $x^{1}=\left(x_{p, 2}^{1}, x_{p, 1}^{1}, x_{q, 1}^{1}, x_{k, 1}^{1}\right)=$ $(9,-1.5,1.25,-3.75)$. Similar to the above processes, $E C\left((2,0,0), C, x^{1}\right)=1$, $E C\left((1,0,0), C, x^{1}\right)=0.5, E C\left((0,1,0), C, x^{1}\right)=0.75, E C\left((0,0,1), C, x^{1}\right)=0.75$. Вy Equation (1),

$$
\begin{aligned}
x_{p, 2}^{2} & =h_{p, 2}\left(x^{1}\right) \\
& =x_{p, 2}^{1}+t \cdot\left[\left(E C\left((2,0,0), C, x^{1}\right)-E C\left((0,1,0), C, x^{1}\right)\right)+\left(E C\left((2,0,0), C, x^{1}\right)-E C\left((0,0,1), C, x^{1}\right)\right)\right] \\
& =9+\frac{1}{4} \cdot[(1-0.75)+(1-0.75)] \\
& =9.125
\end{aligned}
$$


$x_{p, 1}^{2}=-1.625, x_{q, 1}^{2}=1.25, x_{k, 1}^{2}=-3.75$, that is, $x^{2}=\left(x_{p, 2}^{2}, x_{p, 1}^{2}, x_{q, 1}^{2}, x_{k, 1}^{2}\right)=$ $(9.125,-1.625,1.25,-3.75)$. By continuing in the above processes, we have that $x^{8}=$ $(9.25,-1.75,1.25,-3.75)=\bar{\gamma}(F, b, C)$.

\section{Concluding Remarks}

1. This article would like to build on the axiomatic results of the MLII. In summary, the purpose of this article is to introduce dynamic analysis for the MLII.

- A dynamic procedure for the MLII is proposed under relative symmetry by focusing on both the participating elements and their active levels. This dynamic outcome is introduced initially.

- Any level complement combination can be continuously adjusted by means of this dynamic mechanism, and it will gradually approach the MLII, reaching its limit.

- The dynamic outcome of this article could be applied to evaluate combinations of the reacting processes. This application does not appear in existing studies.

- Dynamic procedures on traditional circumstances have only considered nonparticipation or participation. However, it is reasonable that each element might take different active levels to operate. Therefore, different from related dynamic procedures on traditional circumstances, this article introduces the dynamic outcome under multi-choice consideration.

2. The main outcome of this article also led to the following motivation:

- Is it possible to propose different types of dynamic processes by analyzing different interactive behaviors?

This is a topic worth discussing in the future.

Author Contributions: A short paragraph specifying the individual distinctions of the authors is as follows. Conceptualization, J.-C.H., K.H.-C.C. and Y.-H.L.; methodology, Y.-H.L.; validation, Y.-H.L.; formal analysis, Y.-H.L.; investigation, J.-C.H., K.H.-C.C. and Y.-H.L.; writing-original draft preparation, Y.-H.L.; writing - review and editing, J.-C.H. and K.H.-C.C. All authors have read and agreed to the published version of the manuscript.

Funding: This study was funded by National Pingtung University NPTU-110-003.

Institutional Review Board Statement: Not applicable.

Informed Consent Statement: Not applicable.

Data Availability Statement: Data available on request due to privacy restrictions. The data presented in this study are available on request from the corresponding author.

Acknowledgments: The authors are very grateful to the Editors and anonymous referees for patience, assistance, suggestions and comments which much improve the paper.

Conflicts of Interest: The author declare no conflict of interest.

\section{References}

1. Shapley, L.S. A value for $n$-person game. In Distinctions to the Theory of Games II; Kuhn, H.W., Cker, A.W., Eds.; Princeton Press: Princeton, NJ, USA, 1953; pp. 307-317.

2. Hart, S.; Mas-Colell, A. Potential, value and consistency. Econometrica 1989, 57, 589-614. [CrossRef]

3. Maschler, M.; Owen, G. The consistent Shapley value for hyperplane games. Int. J. Game Theory 1989, 18, 389-407. [CrossRef]

4. $\quad$ Ransmeier, J.S. The Tennessee Valley Authority; Vanderbilt University Press: Nashville, TN, USA, 1942.

5. Moulin, H. The separability axiom and equal-sharing methods. J. Econ. Theory 1985, 36, 120-148. [CrossRef]

6. Stearns, R.E. Convergent transfer schemes for n-person games. Trans. Am. Math. Soc. 1968, 134, 449-459.

7. Hwang, Y.-A. An NTU value under complement reduced game. Int. J. Game Theory 2009, 38, 305-324. [CrossRef]

8. Billera, L.J. Global stability in n-person games. Trans. Am. Math. Soc. 1972, 172, 45-56.

9. Hwang, Y.-A.; Liao, Y.-H. The consistent value of fuzzy games. Fuzzy Sets Syst. 2009, 160, 644-656. [CrossRef]

10. Abuteen, E.; Freihat, A.; Al-Smadi, M.; Khalil, H.; Khan, R.A. Approximate series solution of nonlinear, fractional Klein-Gordon equations using fractional reduced differential transform method. J. Math. Stat. 2016, 12, 23-33. [CrossRef] 
11. Al-Smadi, M. Simplified iterative reproducing kernel method for handling time-fractional BVPs with error estimation. Ain Shams Eng. J. 2018, 9, 2517-2525. [CrossRef]

12. Freihet, A.; Hasan, S.; Al-Smadi, M.; Gaith, M.; Momani, S. Construction of fractional power series solutions to fractional stiff system using residual functions algorithm. Adv. Differ. Equa. 2019, 95. [CrossRef]

13. Chen, K.H.-C.; Huang, J.-C.; Liao, Y.-H. Sustainable combination mechanism for catalysts: A game-theoretical approach. Catalysts 2021, 11, 345. [CrossRef]

14. Habschied, K.; Kanižai Šarić, G.; Krstanović, V.; Mastanjević, K. Mycotoxins-Biomonitoring and Human Exposure. Toxins 2021, 13, 113. [CrossRef] [PubMed]

15. Mouchbahani-Constance, S.; Sharif-Naeini, R. Proteomic and Transcriptomic Techniques to Decipher the Molecular Evolution of Venoms. Toxins 2021, 13, 154. [CrossRef] [PubMed]

16. Peles, F.; Sipos, P.; Kovács, S.; Győri, Z.; Pócsi, I.; Pusztahelyi, T. Biological Control and Mitigation of Aflatoxin Contamination in Commodities. Toxins 2021, 13, 104. [CrossRef] [PubMed]

17. Reichwaldt, E.S.; Stone, D.; Barrington, D.J.; Sinang, S.C.; Ghadouani, A. Development of Toxicological Risk Assessment Models for Acute and Chronic Exposure to Pollutants. Toxins 2016, 8, 251. [CrossRef] [PubMed]

18. Sotnichenko, A.; Pantsov, E.; Shinkarev, D.; Okhanov, V. Hydrophobized Reversed-Phase Adsorbent for Protection of Dairy Cattle against Lipophilic Toxins from Diet. Efficiensy In Vitro and In Vivo. Toxins 2019, 11, 256. [CrossRef] [PubMed] 